\title{
Gait variability as digital biomarker of disease severity in Huntington's disease
}

\author{
Heiko Gaßner ${ }^{1}$ - Dennis Jensen ${ }^{1}$. F. Marxreiter ${ }^{1} \cdot$ Anja Kletsch $^{2}$ - Stefan Bohlen ${ }^{2} \cdot$ Robin Schubert $^{2}$. \\ Lisa M. Muratori ${ }^{2,3} \cdot$ Bjoern Eskofier $^{4}$. Jochen Klucken ${ }^{1,5,6} \cdot$ Jürgen Winkler $^{1} \cdot$ Ralf Reilmann $^{2,7,8} \cdot$ Zacharias Kohl $^{1,9,10}$
}

Received: 20 September 2019 / Revised: 20 January 2020 / Accepted: 22 January 2020 / Published online: 11 February 2020

(c) The Author(s) 2020

\begin{abstract}
Background Impaired gait plays an important role for quality of life in patients with Huntington's disease (HD). Measuring objective gait parameters in HD might provide an unbiased assessment of motor deficits in order to determine potential beneficial effects of future treatments.

Objective To objectively identify characteristic features of gait in HD patients using sensor-based gait analysis. Particularly, gait parameters were correlated to the Unified Huntington's Disease Rating Scale, total motor score (TMS), and total functional capacity (TFC).

Methods Patients with manifest HD at two German sites $(n=43)$ were included and clinically assessed during their annual ENROLL-HD visit. In addition, patients with HD and a cohort of age- and gender-matched controls performed a defined gait test $(4 \times 10 \mathrm{~m}$ walk). Gait patterns were recorded by inertial sensors attached to both shoes. Machine learning algorithms were applied to calculate spatio-temporal gait parameters and gait variability expressed as coefficient of variance (CV).

Results Stride length $(-15 \%)$ and gait velocity $(-19 \%)$ were reduced, while stride $(+7 \%)$ and stance time $(+2 \%)$ were increased in patients with HD. However, parameters reflecting gait variability were substantially altered in HD patients (+ $+17 \%$ stride length CV up to $+41 \%$ stride time CV with largest effect size) and showed strong correlations to TMS and TFC $\left(0.416 \leq r_{\mathrm{Sp}} \leq 0.690\right)$. Objective gait variability parameters correlated with disease stage based upon TFC.

Conclusions Sensor-based gait variability parameters were identified as clinically most relevant digital biomarker for gait impairment in HD. Altered gait variability represents characteristic irregularity of gait in HD and reflects disease severity.
\end{abstract}

Keywords Huntington's disease · Gait analysis · Wearable sensors · Gait variability · Regularity of gait

Electronic supplementary material The online version of this article (https://doi.org/10.1007/s00415-020-09725-3) contains supplementary material, which is available to authorized users.

Zacharias Kohl

Zacharias.Kohl@klinik.uni-regensburg.de

1 Department of Molecular Neurology, University Hospital Erlangen, Friedrich-Alexander University Erlangen-

Nürnberg (FAU), Schwabachanlage 6, 91054 Erlangen, Germany

2 George-Huntington Institute (GHI) GmbH, Münster, Germany

3 Rehabilitation Research and Movement Performance Laboratory (RRAMP Lab), Stony Brook University, Stony Brook, NY, USA

4 Machine Learning and Data Analytics Lab, Friedrich-Alexander University Erlangen-Nürnberg (FAU), Erlangen, Germany
5 Medical Valley-Digital Health Application Center GmbH, Bamberg, Germany

6 Fraunhofer Institute for Integrated Circuits IIS, Erlangen, Germany

7 Department of Radiology, University of Muenster, Muenster, Germany

8 Department of Neurodegenerative Diseases and Hertie-Institute for Clinical Brain Research, University of Tuebingen, Tuebingen, Germany

9 Center for Rare Diseases Erlangen, University Hospital Erlangen, Erlangen, Germany

10 Department of Neurology, University of Regensburg, Regensburg, Germany 


\section{Introduction}

Huntington's disease (HD) is an autosomal-dominant, neurodegenerative disease characterized by the triad of motor deficits, cognitive decline, and neurobehavioral symptoms [1]. In particular, gait impairment plays an important role upon motor functioning as it affects the quality of life, limits the independence of patients with $\mathrm{HD}$, and reduces activities of daily living [2]. From a biomechanical viewpoint, gait is a well-defined movement in humans including regular, cyclic-repetitive sequences [3] making it an ideal kinetic process to be analyzed by instrumented measures. In one of the initial detailed descriptions of HD, Osler noted a "curious irregular gait" [4] without specifying the HD gait irregularity in detail. Measuring gait parameters in patients with HD supports a quantitative and unbiased assessment of motor deficits and provides objective measures to quantify potential beneficial effects of future treatments. Objective gait parameters may provide metric, granular information complementing the Unified Huntington's disease Rating Scale total motor score (TMS), and total functional capacity (TFC) as established instruments in daily clinical routine evaluating motor impairment and functionality in HD [5].

Irregularity of gait in HD may be evaluated by using objective measures from instrumented gait analysis systems that are able to calculate stride-by-stride variance, in contrast to, e.g. stopwatch measures focusing on mean values. It has been shown that patients with HD walk slower and with smaller steps compared to healthy controls [6-8]. Recent studies using instrumented carpets or $3 \mathrm{~d}$ motion capture reported results indicating correlations between UHDRS TMS and stride length in a small cohort $(n=7)$ [9] and significant angular changes of the gait cycle $(n=30)$ [10]. Gait variability as a measure of regularity of gait and dynamic postural control seems to be increased in $\operatorname{HD}[8,9,11,12]$. However, these monocentric studies should be interpreted with caution due to small cohorts examined.

Wearable sensors (accelerometers and gyroscopes) combined with machine learning algorithms have shown to provide objective, granular measures that support the rather rater- and time-dependent clinical ratings in neurologic diseases such as Parkinson's disease [13-16]. Moreover, inertial sensors have the potential to be used in long-term monitoring scenarios at the patients' home under real-life conditions with the advantage to record gait patterns over several hours instead of very short-lasting periods during an outpatient visit [17-19]. In HD, it has been shown that accelerometer-based sensors differentiate between pre-manifest and manifest HD patients in a cohort of 14 subjects [6]. In particular, sensor-derived velocity, step and stride length were reduced in manifest HD patients. Moreover, machine learning algorithms provide a framework for gait classification to distinguish HD patients from healthy controls [20]. These mobile sensor technologies combined with intelligent algorithms may support the diagnostic workup. However, the clinical relevance of objective parameters provided by wearable systems in comparison to data gained in well-established clinical scores as UHDRS TMS and TFC of HD patients has not been evaluated so far. Furthermore, gait characteristics in HD, particularly irregularity of gait, need to be understood in more depth by clinical validation. The identification of objective sensor-based gait parameters most characteristic for HD gait and reflecting disease severity is still pending.

To address these questions, the aim of the present twocenter approach was to objectively assess characteristic features of gait in $43 \mathrm{HD}$ patients compared to age- and gendermatched controls using mobile sensor-based gait analysis. In particular, these gait parameters were correlated to the clinical scores TMS and TFC in order to understand whether objective measures reflect disease severity assessed by clinical rating scales.

\section{Subjects and methods}

Fifty patients with manifest HD were enrolled at two German sites, the Department of Molecular Neurology at the University Hospital Erlangen and the George-Huntington Institute (GHI) $\mathrm{GmbH}$, Münster. HD patients received standardized clinical assessments during their annual Enroll-HD visit including UHDRS-TMS and TFC. Enroll-HD is a worldwide observational study monitoring symptoms and disease progression over time in manifested HD patients or patients at-risk (www.enroll-hd.org). In addition, patients with HD and a cohort of age- and gender matched healthy

Table 1 Characteristics of patients with HD and controls (mean \pm SD)

\begin{tabular}{llll}
\hline & HD & Controls & $p$ \\
\hline$n$ & 43 & 43 & \\
Age (years) & $50.0 \pm 11.1$ & $51.0 \pm 11.3$ & 0.653 \\
Gender (m:f) & $25: 18$ & $21: 22$ & $0.387^{\dagger}$ \\
Weight $(\mathrm{kg})$ & $78.7 \pm 20.0$ & $76.9 \pm 16.4$ & 0.658 \\
Height $(\mathrm{cm})$ & $173.9 \pm 9.0$ & $173.7 \pm 9.5$ & 0.935 \\
CAG repeats & $44.1 \pm 4.2(n=40)$ & - & - \\
UHDRS TMS & $38.2 \pm 17.9$ & - & - \\
TFC score & $9.1 \pm 3.4$ & - & - \\
MMSE & $27.1 \pm 3.2(n=40)$ & - & - \\
\hline
\end{tabular}

Mann-Whitney $U$ test. Significance level $p<0.05$

${ }^{\dagger}$ Chi square test

CAG repeats cytosine-adenine-guanine repeats, UHDRS TMS Unified Huntington's Disease Rating Scale total motor score, TFC total functional capacity, MMSE mini-mental state examination 
controls performed a standardized $4 \times 10 \mathrm{~m}$ walk test [21] In order to investigate HD patients with manifest motor symptoms solely (TMS $>5$ ), HD patients with TMS $\leq 5$ (absence of motor symptoms) were excluded $(n=5)$, two datasets failed due to technical reasons. Thus, 43 datasets were analyzed and compared to 43 controls (Table 1). In 40 HD patients, genetic testing revealed increased Cytosin, Adenin and Guanin (CAG)-trinucleotide expansion. In the remaining patients ( $n=3$ ), clinically manifest HD symptoms and positive family history were present; however, genetic testing was not performed.

Gait characteristics were evaluated in a standardized gait test using an instrumented, sensor-based gait analysis system. This system consists of wearable SHIMMER sensors (Shimmer Research Ltd., Dublin, Ireland) laterally attached to the posterior portion of both shoes (Supplementary Figure S1) [22]. Gait signals were recorded within a (tri-axial) accelerometer range of $\pm 6 \mathrm{~g}$ (sensitivity $300 \mathrm{mV} / \mathrm{g}$ ), a gyroscope range of $\pm 500 \% \mathrm{sec}$ (sensitivity $2 \mathrm{mV} / \mathrm{degree} / \mathrm{sec}$ ), and a sampling rate of $102.4 \mathrm{~Hz}$. Sensor signals were transmitted to a tablet computer via Bluetooth ${ }^{\circledR}$ and stored for subsequent data analysis [13, 23]. Machine learning algorithms were applied to calculate spatio-temporal gait parameters as mean per stride values derived from $4 \times 10 \mathrm{~m}$ gait tests (e.g. stride length, gait velocity) [21, 24]. Gait variability as a measure of stride-by-stride variance is presented as the coefficient of variance $(\mathrm{CV})$ of each parameter using an average of 40 strides per patient. Participants performed a standardized $4 \times 10 \mathrm{~m}$ overground gait test on a $10 \mathrm{~m}$-long corridor at both study sites in self-selected walking speed and without stops at turning points. Only straight strides were automatically detected by the stride detection algorithm [21] and used for gait parameter calculations as described [24].

The study was approved by the local ethics committees (IRB-No. 4208, 21.04.2010, amendment approved 06.02.2017, Medical Faculty, Friedrich-Alexander University Erlangen-Nürnberg (FAU), Germany, and IRB-No. 2017-079-f-S, 05.07.2017, Medical Council WestfalenLippe and Westfälische Wilhelms-Universität Münster, Germany). All participants signed the written informed consent according to the Declaration of Helsinki.

\section{Statistical analysis}

Normality of data was tested by Shapiro-Wilk test and variance homogeneity by Levene test. Mann-Whitney $U$ test was used to verify group differences regarding anthropometric variables (age, weight, and height). Gender differences between groups were evaluated using Chi-squared test. Since gait parameters were not distributed normally, Mann-Whitney $U$ test and Kruskal-Wallis test (for group comparisons with TFC) were used to identify group differences in gait characteristics. Kruskal-Wallis test was followed by Dunn-Bonferroni post-hoc tests in order to analyze separate differences between the groups. Besides $p$ values (significance level $\alpha=0.05$ ), Cohens $d$ is presented as measure of effect size. Correlation analysis between gait parameters and clinical scores (UHDRS TMS and TFC) was performed using Spearman's rank correlation $\left(r_{\mathrm{Sp}}\right)$. Moreover, gait characteristics were compared in TFC groups based on a previously described classification [25]. TFC scores 11-13 were grouped in stage I (early), 7-10 stage II (moderate), and 0-6 stage III (advanced). Due to small sample sizes in severely affected HD patients (TFC scores 1-2 and 0 ), patients with TFC scores from 0 to 6 were combined in one category (III).

\section{Results}

\section{Between-group differences in gait parameters}

Specific gait parameters such as stride length (mean \pm SD; HD: $1.30 \pm 0.25 \mathrm{~m}$, controls: $1.52 \pm 0.12 \mathrm{~m}$, delta $\Delta=-15 \%$, $p<0.000$ ) and gait velocity (HD: $1.20 \pm 0.29 \mathrm{~m} / \mathrm{s}$, controls: $1.48 \pm 0.16 \mathrm{~m} / \mathrm{s}, \Delta=-19 \%, p<0.000$ ) were severely reduced, as expected. Stride time (HD: $1.11 \pm 0.15 \mathrm{~s}$, controls: $1.03 \pm 0.08 \mathrm{~s}, \Delta=+7 \%, p=0.008)$ and stance time (HD: $64.5 \pm 2.55 \%$, controls: $63.4 \pm 1.16 \%, \Delta=+2 \%$, $p=0.045$ ) were significantly increased in patients with HD compared to controls (Fig. 1).

Group differences for parameters representing irregularity of gait were more pronounced in HD in comparison to controls: stride time CV (HD: $4.46 \pm 1.55 \%$, controls: $2.80 \pm 0.81 \%, \Delta=+37 \%, p<0.000$ ), swing time CV (HD: $5.59 \pm 2.51 \%$, controls: $3.28 \pm 1.43 \%, \Delta=+41 \%, p<0.000$ ), stance time CV (HD: $3.06 \pm 1.26 \%$, controls: $1.91 \pm 0.91 \%$, $\Delta=+38 \%, p<0.000$ ), stride length CV (HD: $7.96 \pm 2.13 \%$, controls: $6.59 \pm 3.08 \%, \Delta=+17 \%, p=0.001)$, and gait velocity CV (HD: $8.79 \pm 2.43 \%$, controls: $7.39 \pm 2.84 \%$, $\Delta=+16 \%, p=0.001$ ) (Fig. 2). Cohen's $d$ effect sizes showed the largest differences between groups for the gait variability parameter stride time CV (Cohen's $d=1.345$ ), followed by swing time CV $(d=1.129)$, and stance time CV $(d=1.040)$. The effect size for stride length $(d=1.149)$ and gait velocity $(d=1.212)$ was strong but did not reach those of stride time CV (Supplementary Table T1).

\section{Correlation analysis between gait parameters and clinical scores}

Parameters representing gait variability showed moderate to strong correlations to UHDRS TMS: stride time $\mathrm{CV}\left(r_{\mathrm{Sp}}=0.676, p \leq 0.000\right)$, stance time $\mathrm{CV}\left(r_{\mathrm{Sp}}=0.690\right.$, $p \leq 0.000)$, swing time $\mathrm{CV}\left(r_{\mathrm{Sp}}=0.595, p \leq 0.000\right)$, stride 
Fig. 1 Spatio-temporal gait parameters: stride length $(\mathrm{cm})$, gait velocity $(\mathrm{m} / \mathrm{s})$, stance time $(\%)$, and swing time (\%) show significant differences between patients with $\mathrm{HD}$ and controls $(* p<0.05 ; * * * p<0.001)$
A

Stride time CV (\%)

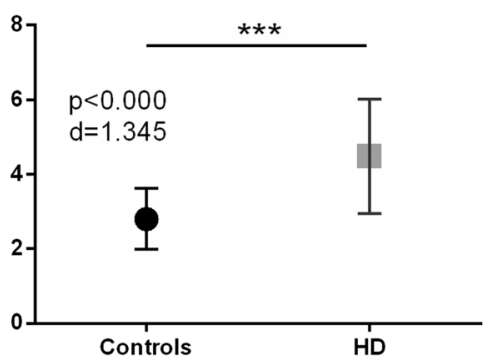

B

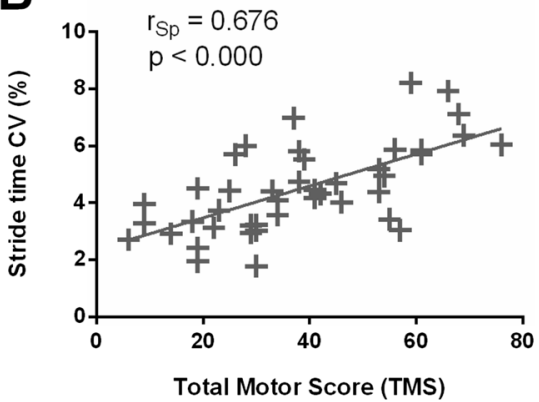

Stride length $(\mathrm{cm})$

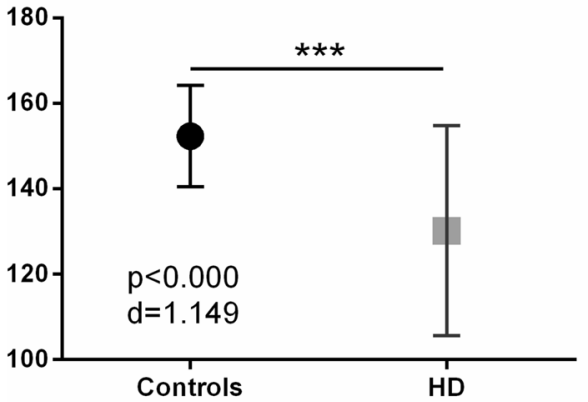

Stance time $(\%)$

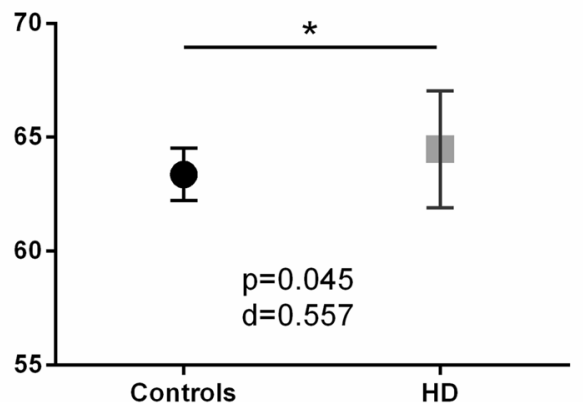

Stance time CV (\%)
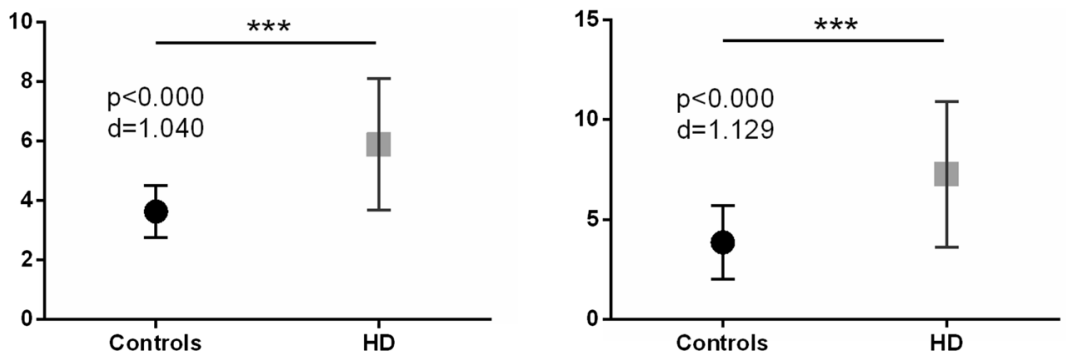

Gait velocity $(\mathrm{m} / \mathrm{s})$

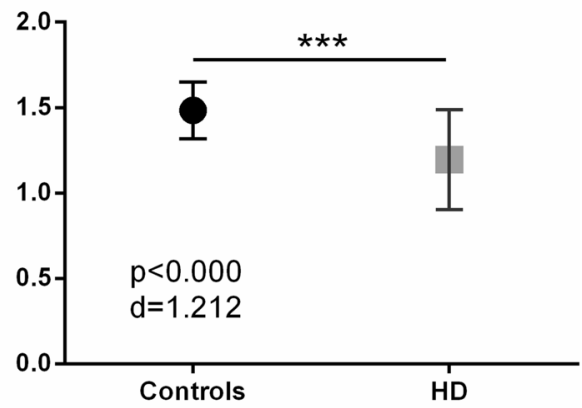

Swing time (\%)

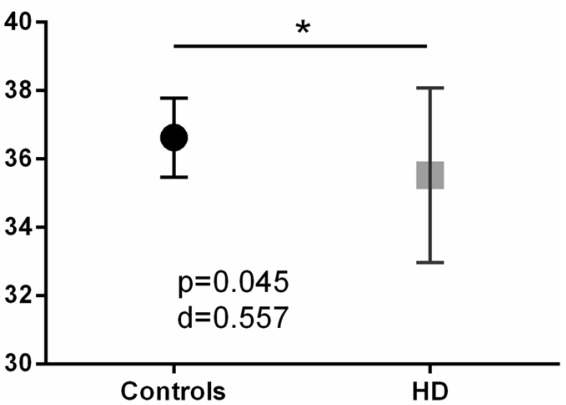

Fig. 2 a Variability in stride time, stance time and swing time is significantly increased in patients with HD compared to healthy controls $(* * * p<0.001) . d$ Cohen's $d$ effect size, $C V$ coefficient of variance. $\mathbf{b}$
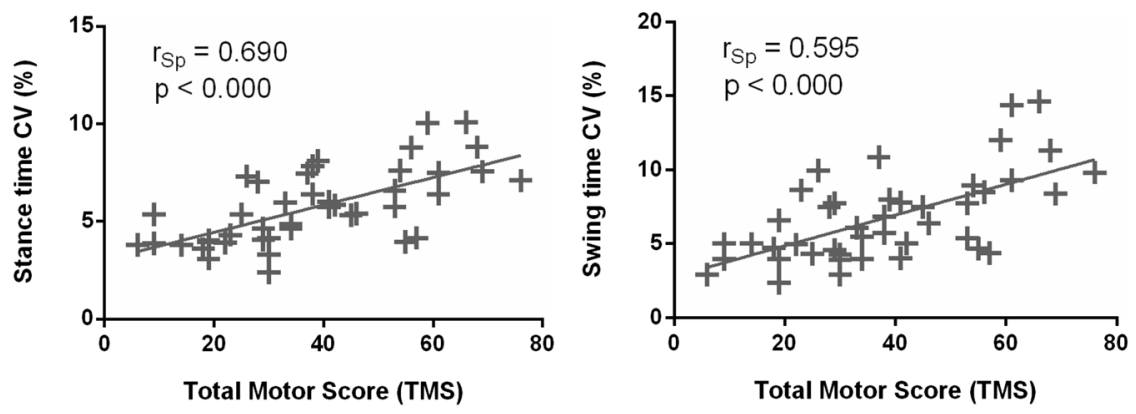

Stride time CV, stance time CV, and swing time CV of patients with HD correlate to UHDRS total motor score, $r_{S p}$ Spearman's rank correlation coefficient 
length $\mathrm{CV}\left(r_{\mathrm{Sp}}=0.416, p=0.006\right)$, and gait velocity $\mathrm{CV}$ $\left(r_{\mathrm{Sp}}=0.579, p \leq 0.000\right)$. Stride length and gait velocity showed moderate inverse correlations to UHDRS TMS: stride length $\left(r_{\mathrm{Sp}}=-0.549, p \leq 0.000\right)$, and gait velocity $\left(r_{\mathrm{Sp}}=-0.478, p=0.001\right)$.

Furthermore, the objective gait variability measures reflected the patients' functional abilities according to TFC by moderate inverse correlations: stride time CV $\left(r_{\mathrm{Sp}}=-0.555, p \leq 0.000\right)$, stance time CV $\left(r_{\mathrm{Sp}}=-0.521\right.$, $p \leq 0.000)$, swing time $\mathrm{CV}\left(r_{\mathrm{Sp}}=-0.561, p \leq 0.000\right)$, stride length $\mathrm{CV}\left(r_{\mathrm{Sp}}=-0.468, p=0.002\right)$, and gait velocity $\mathrm{CV}\left(r_{\mathrm{Sp}}=-0.628, p \leq 0.000\right)$. Graphs to this correlation analysis are presented in Figs. 2 and 3. Group comparisons between TFC subgroups (early, moderate, advanced) revealed highly significant differences in stride time $\mathrm{CV}$ $(p<0.001, d=1.601)$, stance time CV $(p=0.002, d=1.214)$, swing time CV $(p=0.001, d=1.347)$, stride length $\mathrm{CV}$ $(p=0.010, d=0.935)$, and gait velocity $\mathrm{CV}(p<0.000$, $d=1.613)$. Dunn-Bonferroni post-hoc tests showed differences between the TFC subgroups for stride time CV (early vs. advanced: $p<0.000$, moderate vs. advanced: $p=0.045$ ), stance time CV (early vs. advanced: $p=0.001$ ), swing time CV (early vs. advanced: $p<0.000$ ), stride length CV (early vs. advanced: $p=0.008$ ), and gait velocity CV (early vs. advanced: $p<0.000$ ) (Fig. 3b). Importantly, stride time CV was the sole parameter detecting differences between moderate and advanced HD patients.

\section{Discussion}

The aim of this study was to objectively assess characteristic features of gait in HD patients compared to age- and gendermatched controls using mobile sensor-based gait analysis. In particular, gait parameters were correlated to clinical scores (TMS and TFC) in order to clinically validate these objective
A

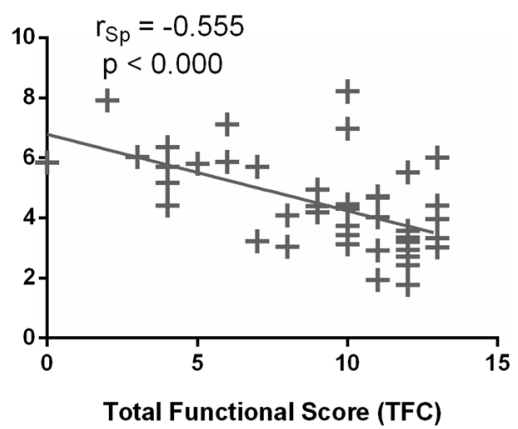

B

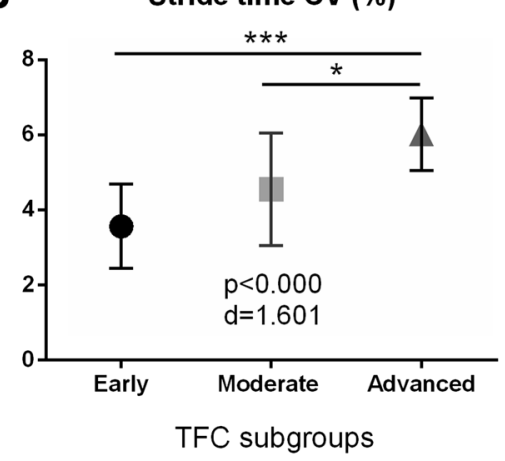

Stance time CV (\%)

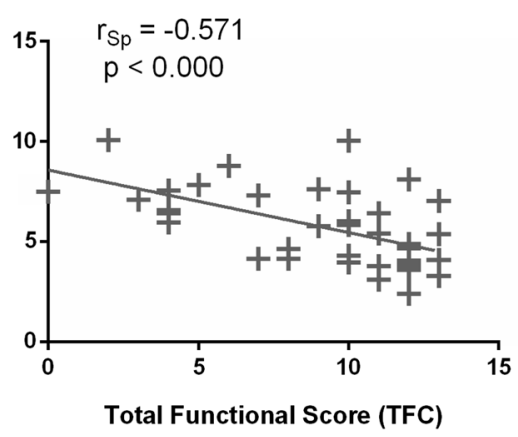

Stance time CV (\%)

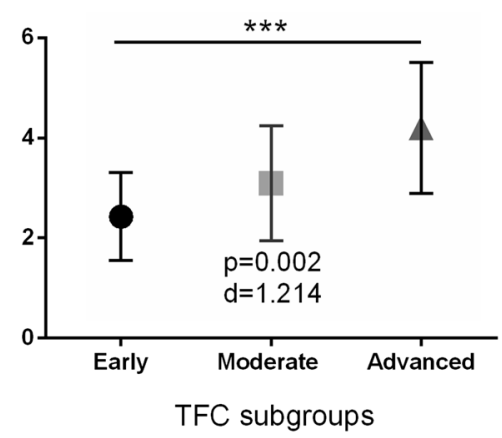

Swing time CV (\%)

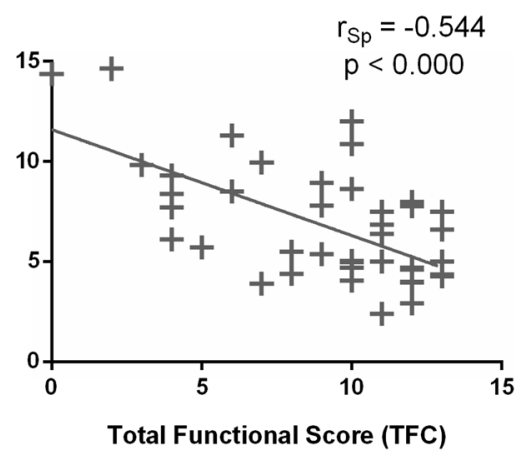

Swing time CV (\%)

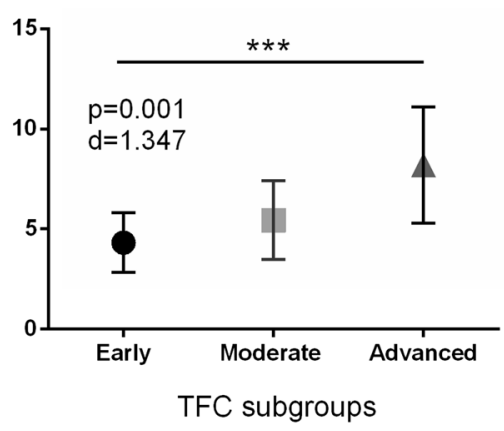

$\begin{array}{ll}\text { Early } & n=19 \\ \text { Moderate } & n=14\end{array}$

- Advanced $n=10$
Fig. 3 a Stride time CV, stance time $\mathrm{CV}$ and swing time $\mathrm{CV}$ of patients with HD correlate to TFC. $C V$ coefficient of variance, $r_{S p}$ Spearman's rank correlation coefficient. b Stride time CV, stance time
$\mathrm{CV}$ and swing time $\mathrm{CV}$ of patients with HD grouped by TFC score $\left({ }^{*} p<0.05 ; * * * p<0.001\right)$. Early $\mathrm{HD}=\mathrm{TFC}$ score $11-13$, moderate $\mathrm{HD}=\mathrm{TFC} 7-10$, advanced HD $=$ TFC $0-6 . d$ Cohen's $d$ effect size 
parameters. The main finding demonstrated that gait variability parameters representing the disease-characteristic irregularity of gait were the most relevant parameters correlating with TMS and TFC. Increased stride time CV in HD patients showed the largest effect size in comparison to gait of controls. Moreover, we observed severely reduced stride length and gait velocity as well as significantly increased stride time, and stance time in HD patients compared to healthy controls, albeit, with smaller effect sizes.

\section{Gait impairment in HD}

Objective gait measures of the present study showed that HD patients walk slower $(-19 \%)$ and with shorter steps $(-15 \%)$ compared to matched healthy controls. Importantly, we observed that gait variability representing the HD-characteristic irregular gait signature is substantially increased in HD patients (17-41\%). Herewith, we confirm previous findings, however with mobile, sensor-based technology in comparison to stationary systems [12]. Gait variability is serving as a quantitative marker for quality of gait and falls $[8,11,26]$. With regard to stride length and gait velocity, we confirm results from previous small-cohort studies reporting motor impairment reflected by gait parameters, however with more statistical power due to larger sample sizes in our dual-center study with age- and gender-matched cohorts. Intriguingly, we confirm by applying mobile sensor-based technology that especially gait variability parameters characterize at best HD-typical gait. The largest effect size for differences between gait in HD patients and matched healthy controls was noticed for stride time CV $(d=1.345)$ followed by gait velocity $(d=1.212)$, stride length $(d=1.149)$, swing time CV $(d=1.129)$, and stance time CV $(d=1.040)$. These results suggest that gait parameters derived from sensorbased gait analysis serve as an objective, digital biomarker for gait patterns in HD. In particular, increased gait variability appeared to be characteristic for HD patients. This finding is in line with previous studies showing increased movement variability of the upper extremity by investigating finger tapping, and grasp force in HD [27-30]. Grip force variability is discussed as an objective measure to evaluate motor deficits and reflect disease progression in HD [31]. Our results identify gait variability as a diseasecharacteristic signature and an important digital biomarker in terms of gait dysfunction in HD patients, similar to grip force variability as an objective and quantitative outcome for motor deficits in the upper extremity. In a small longitudinal study with ten pre-manifest gene carriers, gait variability has increased within the first year from baseline and may be a likely marker for disease progression [32]. Future studies should further investigate sensor-based gait variability parameters longitudinally in order to determine whether this measure may be an appropriate progression marker for
HD. In addition, gait variability should be investigated in premanifest HD patients in order to evaluate this measure as potential early-detection marker for clinical symptoms. Previous work supports that it is worth investigating quantitative gait in premanifest HD patients [8].

\section{Sensor-based gait variability parameters reflect disease severity}

Gait variability parameters derived from sensor-based gait analysis strongly correlated to the established clinical motor ratings TMS and TFC. This finding indicates that $\mathrm{CV}$ measures are an objective mirror of the ordinal clinical motor examinations. Instrumented quantitative measures are discussed as digital biomarkers and complementary outcomes since clinical rating scales are limited due to inter- and intrarater variability as well as rater-induced placebo effects in clinical trials [33-35]. A meta-analysis based on 800 patients with Parkinson's disease and 854 healthy subjects provided evidence that a stride time variability larger than $2.4 \%$ discriminates healthy from pathological gait [36]. Sensor-based gait variability parameters were identified as very important objective measure in differentiating patients with atypical parkinsonian disorders (larger gait variability) from patients with sporadic Parkinson's disease [22]. In HD, it has been shown that the instrumented measure of variability in grasp forces strongly correlated with motor performance assessed by TFC $(r=-0.712)$ and TMS $(r=0.841)$ suggesting that movement variability is a key feature of motor impairment in HD [29]. In a similar way, quantitative assessments of chorea in HD patients has shown to be feasible, easy applicable and may improve sensitivity and reliability of motor end points in clinical studies [34]. Therefore, instrumented quantitative data provide important measurements complementing established clinical ratings and may be useful for the evaluation of motor deficits in HD.

\section{Wearable sensor system as easy-to-apply tool for objective gait assessment}

We observed that wearable sensors provide metric, granular information in regard to gait impairment in HD patients. Easy-to-apply wearable systems are able to provide strideby-stride variance parameters which have in this study been demonstrated to play an important role in HD gait. Sensorbased gait data correlated to established clinical scores and, therefore, indicates that inertial sensors are able to reflect the rating of clinical experts. This is in line with previous studies in other basal ganglia diseases like Parkinson's disease [13-15] and atypical Parkinsonian disorders [16] reporting that wearable sensor systems support the clinical workup by objective, quantitative data. In HD, previous studies observed that inertial sensors are able to differentiate 
between HD patients and healthy controls [20]. They have been validated for the analysis of gait characteristics in HD patients by comparing spatio-temporal gait parameters derived from sensors with those from instrumented gait mats [6, 37]. In contrast to mats, future mobile sensor technology may be used in home-monitoring scenarios [17, 18] over several hours in order to provide a comprehensive and long-lasting monitoring tool complementing established short-lasting clinical examinations in the outpatient units. In a pilot study, the feasibility was shown to use wearable sensors in the hospital and in the home-environment of HD patients [38]. Future studies need to further investigate the application of inertial sensors as objective measure to detect motor impairment more precisely and outside the lab. In particular, it is interesting to record irregular movements in the upper and lower extremity of HD patients in everyday life.

\section{Conclusion}

In conclusion, our data demonstrate that sensor-based gait variability parameters were identified as the clinically most relevant digital biomarker for gait impairment in HD. They showed the largest effect size in group comparison and strongly correlated to established clinical scores (TFC, TMS). Thus, sensor-based gait variability represents the irregularity of gait characteristic for HD and reflects disease severity. Moreover, we observed reduced stride length and gait velocity as well as increased stride time and stance time in HD patients compared to age- and gender-matched controls. Our cohort-based clinical validation study confirmed the clinical relevance of a detailed and objective gait analysis in HD. As the altered sensor-based gait variability parameters of this study may mirror disease progression according to TFC, there is a strong need for longitudinal studies validating these initial findings. The transfer from crosssectional studies to disease monitoring including long-term recordings at patients' home and individual care should be the focus of future studies using sensor-based gait analysis.

Acknowledgements Open Access funding provided by Projekt DEAL. The authors would like to thank all patients that participated in this study. The present work is part of the doctoral thesis of Dennis Jensen in the frame of obtaining the degree "Dr. med." at the University Hospital Erlangen, Friedrich-Alexander University Erlangen-Nürnberg (FAU). This study was supported by the Huntington-Stiftung of the Deutsche Huntington Hilfe e.V. We further acknowledge the EnrollHD staff for their support and collaboration. Bjoern Eskofier gratefully acknowledges the support of the German Research Foundation (DFG) within the framework of the Heisenberg professorship programme (Grant Number ES 434/8-1).

Author contributions Conception and design of the study: HG, DJ, SB, JK, RR, ZK. Execution: HG, DJ, FM, AK, SB, RS, LMM, RR, ZK.
Statistical analysis: HG, DJ. Writing the first draft of the manuscript: HG, DJ. Manuscript review and critique: LMM, BE, JK, JW, RR, ZK.

Funding $\mathrm{HG}, \mathrm{BE}, \mathrm{JK}$ and JW received institutional research grants from the Emerging Field Initiative of the Friedrich Alexander-University Erlangen-Nürnberg (EFI Moves, 2 Med 03), from the Bavarian State Ministry for Education, Science and the Arts, Munich, Germany (MotionLab@Home, ElHome Center), and from the Bavarian Ministry of Economic Affairs and Media, Energy and Technology, Germany (Medical Valley Award 2016, Fall Risk PD). HG, FM, RR, and ZK received an institutional research grant by the Huntington-Stiftung of the Deutsche Huntington Hilfe e.V. FM is supported by the Interdisciplinary Center for Clinical Research of the FAU, Clinician Scientist programme. HG, BE, and JK are supported by Mobilise-D from the Innovative Medicines Initiative 2 Joint Undertaking under grant agreement no. 820820 . HG received support by the Medical Research Foundation at the University Hospital Erlangen outside of the submitted work. BE holds ownerships of Portabiles HealthCare Technologies $\mathrm{GmbH}$ and Portabiles $\mathrm{GmbH}$, received compensation and honoraria from serving on scientific advisory boards for Abbvie $\mathrm{GmbH}$, Adidas $\mathrm{GmbH}$, Bosch Sensortec GmbH, and ST Sportservice GmbH. Further, he gratefully acknowledges the support of the German Research Foundation (DFG) within the framework of the Heisenberg professorship programme (grant number ES 434/8-1). JK holds ownerships of Portabiles HealthCare Technologies $\mathrm{GmbH}$ and Portabiles $\mathrm{GmbH}$, received compensation and honoraria from serving on scientific advisory boards for LicherMT GmbH, Abbvie GmbH, UCB Pharma GmbH, GlaxoSmithKline GmbH \& Co. KG, Athenion GmbH, and Thomashilfen $\mathrm{GmbH}$; as well as lecturing from UCB Pharma GmbH, TEVA Pharma $\mathrm{GmbH}$, Licher MT GmbH, Desitin GmbH, Abbvie GmbH, Solvay Pharmaceuticals, and Ever Neuro Pharma GmbH. JW reports personal fees outside of the submitted work from Desitin Arzneimittel GmbH and Biogen GmbH. ZK reports personal fees outside of the submitted work from Desitin Arzneimittel GmbH and UCB Pharma.

\section{Compliance with ethical standards}

Conflicts of interest DJ, AK, SB, LM, and RR declare no conflict of interest.

Ethical standards This study has been approved by the local ethics committees in Erlangen, Germany and Münster, Germany and has, therefore, been performed in accordance with the ethical standards laid down in the 1964 Declaration of Helsinki and its later amendments.

Disclosure The study was supported and performed at the Department of Molecular Neurology (University Hospital Erlangen, Erlangen, Germany), and the George-Huntington Institute (GHI) GmbH, Münster, Germany.

Open Access This article is licensed under a Creative Commons Attribution 4.0 International License, which permits use, sharing, adaptation, distribution and reproduction in any medium or format, as long as you give appropriate credit to the original author(s) and the source, provide a link to the Creative Commons licence, and indicate if changes were made. The images or other third party material in this article are included in the article's Creative Commons licence, unless indicated otherwise in a credit line to the material. If material is not included in the article's Creative Commons licence and your intended use is not permitted by statutory regulation or exceeds the permitted use, you will need to obtain permission directly from the copyright holder. To view a copy of this licence, visit http://creativecommons.org/licenses/by/4.0/. 


\section{References}

1. Walker FO (2007) Huntington's disease. Lancet 369(9557):218-228

2. Kirkwood SC et al (2001) Progression of symptoms in the early and middle stages of Huntington disease. Arch Neurol 58(2):273-278

3. Rau G, Disselhorst-Klug C, Schmidt R (2000) Movement biomechanics goes upwards: from the leg to the arm. J Biomech 33(10):1207-1216

4. Osler W (1894) Case of hereditary chorea. Johns Hopkins Hosp Bull 5:119-129

5. Huntington Study Group (1996) Unified Huntington's disease rating scale: reliability and consistency. Mov Disord 11(2):136-142

6. Dalton A et al (2013) Analysis of gait and balance through a single triaxial accelerometer in presymptomatic and symptomatic Huntington's disease. Gait Posture 37(1):49-54

7. Delval A et al (2006) Role of hypokinesia and bradykinesia in gait disturbances in Huntington's disease. J Neurol 253(1):73-80

8. Rao AK et al (2008) Spectrum of gait impairments in presymptomatic and symptomatic Huntington's disease. Mov Disord 23(8):1100-1107

9. Pyo SJ et al (2017) Quantitative gait analysis in patients with Huntington's disease. J Mov Disord 10(3):140-144

10. Mirek E et al (2017) Three-dimensional trunk and lower limbs characteristics during gait in patients with Huntington's disease. Front Neurosci 11:566

11. Hausdorff JM et al (1998) Gait variability and basal ganglia disorders: Stride-to-stride variations of gait cycle timing in Parkinson's disease and Huntington's disease. Mov Disord 13(3):428-437

12. Moon $Y$ et al (2016) Gait variability in people with neurological disorders: a systematic review and meta-analysis. Hum Mov Sci 47:197-208

13. Klucken $\mathrm{J}$ et al (2013) Unbiased and mobile gait analysis detects motor impairment in Parkinson's disease. PLoS ONE 8(2):e56956

14. Schlachetzki JCM et al (2017) Wearable sensors objectively measure gait parameters in Parkinson's disease. PLoS ONE 12(10): 0183989

15. Marxreiter F et al (2018) Sensor-based gait analysis of individualized improvement during apomorphine titration in Parkinson's disease. J Neurol 265(11):2656-2665

16. Raccagni $C$ et al (2018) Sensor-based gait analysis in atypical parkinsonian disorders. Brain Behav 8(6):e00977

17. Pasluosta CF et al (2015) An emerging era in the management of Parkinson's disease: wearable technologies and the internet of things. IEEE J Biomed Health Inform 19(6):1873-1881

18. Maetzler W, Klucken J, Horne M (2016) A clinical view on the development of technology-based tools in managing Parkinson's disease. Mov Disord 31(9):1263-1271

19. Espay AJ et al (2016) Technology in Parkinson's disease: challenges and opportunities. Mov Disord 31(9):1272-1282

20. Mannini A et al (2016) A machine learning framework for gait classification using inertial sensors: application to elderly, poststroke and Huntington's disease patients. Sensors 16(1):134. https ://doi.org/10.3390/s16010134
21. Barth J et al (2015) Stride segmentation during free walk movements using multi-dimensional subsequence dynamic time warping on inertial sensor data. Sensors (Basel) 15(3):6419-6440

22. Gassner $\mathrm{H}$ et al (2019) The diagnostic scope of sensor-based gait analysis in atypical Parkinsonism: further observations. Front Neurol 10:5

23. Gassner H et al (2017) Gait and cognition in Parkinson's disease: cognitive impairment is inadequately reflected by gait performance during dual task. Front Neurol 8:550

24. Rampp A et al (2015) Inertial sensor-based stride parameter calculation from gait sequences in geriatric patients. IEEE Trans Biomed Eng 62(4):1089-1097

25. Paulsen JS et al (2010) Challenges assessing clinical endpoints in early Huntington disease. Mov Disord 25(15):2595-2603

26. Hausdorff JM (2007) Gait dynamics, fractals and falls: finding meaning in the stride-to-stride fluctuations of human walking. Hum Mov Sci 26(4):555-589

27. Bechtel $\mathrm{N}$ et al (2010) Tapping linked to function and structure in premanifest and symptomatic Huntington disease. Neurology 75(24):2150-2160 (e-Pub ahead of print)

28. Reilmann R et al (2010) Grasping premanifest Huntington's disease-shaping new endpoints for new trials. Mov Disord 25(16):2858-2862

29. Gordon AM et al (2000) Coordination of prehensile forces during precision grip in Huntington's disease. Exp Neurol 163(1):136-148

30. Reilmann R et al (2010) Tongue force analysis assesses motor phenotype in premanifest and symptomatic Huntington's disease. Mov Disord 25(13):2195-2202

31. Reilmann R et al (2001) Objective assessment of progression in Huntington's disease: a 3-year follow-up study. Neurology 57(5):920-924

32. Rao AK et al (2011) Longitudinal change in gait and motor function in pre-manifest Huntington's disease. PLoS Curr 3:RRN1268

33. Reilmann R et al (2012) Huntington's disease: objective assessment of posture-a link between motor and functional deficits. Mov Disord 27(4):555-559

34. Reilmann R et al (2011) Assessment of involuntary choreatic movements in Huntington's disease-toward objective and quantitative measures. Mov Disord 26(12):2267-2273

35. Reilmann R, Schubert R (2017) Motor outcome measures in Huntington disease clinical trials. Handb Clin Neurol 144:209-225

36. König $\mathrm{N}$ et al (2016) Can gait signatures provide quantitative measures for aiding clinical decision-making? A systematic metaanalysis of gait variability behavior in patients with Parkinson's disease. Front Hum Neurosci 10:319

37. Trojaniello D et al (2015) Comparative assessment of different methods for the estimation of gait temporal parameters using a single inertial sensor: application to elderly, post-stroke, Parkinson's disease and Huntington's disease subjects. Gait Posture 42(3):310-316

38. Andrzejewski KL et al (2016) Wearable sensors in Huntington disease: a pilot study. J Huntington's Dis 5(2):199-206 